This document is the accepted manuscript version of the following article:

Allegrini, J., Dorer, V., \& Carmeliet, J. (2014). Buoyant flows in street canyons:

validation of CFD simulations with wind tunnel measurements. Building and Environment, 72, 63-74. http://doi.org/10.1016/j.bui1denv.2013.10.021

This manuscript version is made available under the CC-BY-NC-ND 4.0 1icense

http://creativecommons.org/1icenses/by-nc-nd/4.0/

\title{
Buoyant flows in street canyons: Validation of CFD simulations with wind tunnel measurements
}

\author{
Jonas Allegrini ${ }^{\mathrm{a}, \mathrm{b}^{*}}$, Viktor Dorer ${ }^{\mathrm{a}}$, Jan Carmeliet ${ }^{\mathrm{a}, \mathrm{b}}$ \\ ${ }^{a}$ Laboratory for Building Science and Technology \\ Swiss Federal Laboratories for Materials Science and Technology (Empa) \\ Überlandstrasse 129, 8600 Dübendorf, Switzerland \\ ${ }^{\mathrm{b}}$ Chair of Building Physics, Swiss Federal Institute of Technology Zurich (ETHZ), \\ Wolfgang-Pauli-Strasse 15, 8093 Zürich, Switzerland
}

\begin{abstract}
:
Computational fluid dynamics (CFD) is often used to predict flow structures in urban areas for the determination of pollutant dispersion, human comfort or heat fluxes. During daytime building façades and ground surfaces are heated by solar radiation and thereby induce buoyancy, which changes the flow field around buildings significantly. The CFD models used to simulate buoyant flow fields in urban areas are not sufficiently validated. This study aims to validate CFD simulations for buoyant flows in urban street canyons by comparison with wind tunnel measurements. 2D steady RANS (Reynolds-Averaged Navier-Stokes) CFD simulations were conducted with different near-wall treatments. Velocity, turbulent kinetic energy and temperature profiles from CFD were compared with the measured flow fields (measurement technique: particle image velocimetry). Isothermal cases as well as cases with leeward wall or windward wall heating or all surfaces heated were considered. The results show that CFD can predict the general flow structures and the influence of buoyancy. The detailed flow field inside the street canyon is strongly dependent on the flow structure within the shear layer at the top of the street canyon. Therefore to get accurate results for the flow profiles inside the street canyon, the flow within the shear layer has to be predicted correctly.
\end{abstract}

\section{Keywords}

Buoyancy, Wind tunnel, CFD, Street canyon, Validation, RANS

Corresponding author: Jonas Allegrini, Empa Dübendorf, Ueberlandstrasse 129, 8600 Dübendorf, Switzerland. Tel.: +41 (0) 587656512, Fax: +41 (0) 5876540 09, e-mail: jonas.allegrini@empa.ch

\section{Introduction}

Due to the complexity and scale of the built environment, CFD simulations are often conducted to predict the wind flow in urban areas [1]. CFD studies are used for wind comfort analysis, to access the human comfort at the pedestrian level around buildings (see e.g. [2-4]), for the prediction of pollution dispersion (e.g. [5-8]), to predict the convective heat transfer at building façades in urban areas (e.g. [9,10]), etc. To be able to rely on the CFD results, the simulations need to be validated by measurements. Because it is difficult to measure full-scale urban wind fields at a high enough resolution with controlled boundary conditions, often wind tunnel measurements are conducted for validation purposes. Nowadays RANS simulations with a k- $\varepsilon$ turbulence model are still often applied for urban CFD simulations, due to its computational efficiency, although more advanced and more accurate CFD models exist (e.g. [11-14]). CFD models for flows in urban street canyons are not yet comprehensively validated in literature, especially for cases where buoyancy has an influence on the flow field. Buoyancy in urban street canyons originates from the increased façade temperatures 
due to absorbed longwave and solar radiation. Some validation studies have been conducted for flows in urban street canyons. Kovar-Panskus et al. [15] compared the results of RANS CFD simulations with a standard k- $\varepsilon$ turbulence model with wind tunnel measurements for isothermal street canyons with different aspect ratios. They found a good agreement for the main flow structures, but the CFD simulations tended to underestimate the velocities inside the street canyon. The validation was based on visual comparison of streamlines and vector fields and on comparison of vertical velocity profiles inside the street canyon. Chan et al. [16] conducted CFD simulations (RANS with different k- $\varepsilon$ turbulence model) of pollutant dispersion in a 2D urban street canyon and validated their results with the measurements of Meroney et al. [17]. The flow field was not validated: only the pollution concentrations were compared with the wind tunnel measurements. They found the best agreement occurred when using the RNG model compared to the standard and realizable k- $\varepsilon$ turbulence models. A number of validation studies have also been conducted for street canyons with buoyancy. Memon et al. [18], Xie et al. [19] and Kim and Baik [20,21] compared their CFD results with the measured data of Uehara et al. [22]. For the validation they used velocity and temperature profiles on the vertical centreline of the street canyon. On the centreline the profiles agree rather well for all studies, but a more detailed validation of the total flow field based on parameters such as the exchange rate at the top of the street canyon is needed to be able to rely on the CFD simulations. Xie et al. [23] studied the pollution dispersion in a street canyon with bottom heating. For validation purposes they compared the streamlines of the flow fields with the flow fields measured by Kovar-Panskus et al. [24]. They found that the position of the centre of the vortices agreed reasonably well. Another validation study using the results of Kovar-Panskus et al. [24] was conducted by Tablada and Roels [25]. They conducted RANS simulations with a realizable k- $\varepsilon$ turbulence model for a number of cases that were measured by Kovar-Panskus et al. [24]. Tablada and Roels [25] found an overestimation of the buoyancy effect by the CFD simulations. Garbero et al. [26] conducted a comparison study for a street canyon with windward and leeward wall heating. Due to the rather high freestream velocities there was only one case where buoyancy caused a significant change of the vortex structure. They found a qualitatively good agreement between the measurements and the simulations. A quantitative comparison was not possible because their simulations were conducted for a full-scale street canyon, while the wind tunnel measurements were conducted with a scaled street canyon model. For a cube with leeward wall heating, Dimitrova et al. [27] found that CFD can be useful to predict the flow around a cube with leeward wall heating. Besides validation studies using wind tunnel measurements, studies with full-scale measurement have also been conducted. For example Assimakopoulos et al. [28] conducted such a study for wind directions perpendicular to the street canyon axis and found over- and underestimations of the CFD results compared to the measurements.

Although a number of validation studies have been conducted, there are still further investigations needed, especially for understanding the detailed flow structures of buoyant cases. Most studies found in literature are limited to a visual comparison or to a few profiles at centre position and for a small number of cases. Therefore it is rather difficult to draw a general conclusion from the validation studies reported above. The aim of this study is to conduct a more detailed validation for a wider range of different flow fields in urban street canyons. For the validation, our own measured flow fields [29] are used. We conducted wind tunnel measurements to study the influence of buoyancy on the flow in a scaled street canyon with heated surfaces. The street canyon was elongated over the whole width of the wind tunnel to achieve a high degree of flow two-dimensionality. Therefore in this study the CFD simulations are limited to 2D and conducted with a k- $\varepsilon$ model to save computational costs (RANS). This numerical approach can be found in a number of studies given in literature (e.g. $[10,18,23,30,31])$. Here the aim was to validate this numerical approach rather than to find other numerical models which may give more accurate predictions of the flow fields. Buoyant flows in street canyons have an unsteady and 3D characteristic and therefore more complex numerical models need to be used to get the most accurate flow predictions. The focus of this validation study is on the flow field (velocities and turbulent kinetic energies) (the heat fluxes could not be validated because they were not measured due to the practical difficulties in achieving this [29]). 
The structure of the paper is as follows. In section 2 the experimental setup is briefly discussed. In section 3 the numerical models used for this study are presented. In section 4 the validation methodology is described. The results of the validation are given in section 5 .

\section{Experimental setup}

Our measurements [29] are used for validation purposes. To model the flow in an urban street canyon a cavity of W x H x L (W: width; H: height; L: length) 20 x 20 x $180 \mathrm{~cm}$ was constructed in the wind tunnel (Figure 1). The flow direction was perpendicular to the axis of the cavity. All surfaces of the wind tunnel model were made out of aluminium, which justifies not to model roughness in this validation study. The walls and the ground of the street canyon were heated individually with heating mats (mounted on the back of the aluminium plates). Due to the high thermal conductivity of aluminium, the temperature was almost uniform over the heated surfaces. The flow field was measured with particle image velocimetry (PIV) on a cross-section (near the centre) of the street canyon. The flow fields were measured for isothermal conditions as well as for cases where the walls and the ground surfaces were heated either individually or altogether.

To characterise the flow in terms of buoyancy the Froude number was used in this study:

$$
F r=\frac{U_{F S}^{2}}{g H \frac{T_{w}-T_{r e f}}{T_{r e f}}}
$$

where $U_{F S}$ is the freestream velocity, $T_{w}$ is the surface temperature of the heated wall, $T_{\text {ref }}$ is a reference temperature (here the temperature of the freestream) and $g$ is the gravitational acceleration.

A wide range of Froude numbers was covered by testing at a wide range of surface temperatures and freestream velocities. The different boundary conditions together with the respective Reynolds and Froude numbers are given in Table 1. The temperature at the inflow of the wind tunnel was for all cases $23{ }^{\circ} \mathrm{C}$.

\section{Numerical model}

For this validation study 2D steady RANS CFD simulations using ANSYS Fluent 12.0 [32] were conducted. The standard and the realizable k- $\varepsilon$ models were used to model turbulence. For this study also simulations with other turbulence models (k- $\omega$, Spalart-Allamares, Reynolds stress) were conducted. But in contrast to our expectations they did not perform better for the here studied flows with large vortices. At the near-wall regions the boundary layers were either resolved with low-Reynolds number modelling (LRNM) or modelled with wall functions (WFs).

The dimensions of the 2D computational domain were set according to the wind tunnel dimensions (Figure 2). The height $\mathrm{H}$ of the street canyon was $0.2 \mathrm{~m}$. To carry out a validation study, the CFD results have to be grid independent [33]. Therefore the mesh was built based on a grid sensitivity analysis and on guidelines of Franke et al. [34]. When comparing the results from the simulations of the last grid refinement step, we found that the differences are by order of magnitudes smaller than the differences caused by using the different numerical models presented in this paper. Therefore the conclusions of this paper are not dependent on the used grid. The 2D mesh consisted of 5500 cells. It was refined towards the walls and had maximum $\mathrm{y}+$ values of 4.5. The same mesh was used for simulations with LRNM and for simulations with WFs, resulting in too low $\mathrm{y}+$ values for the simulations with WFs (the consequences of this selection are discussed in the following section). A mesh with high enough $y+$ values for WFs could not be used, because the mesh would be too coarse (width of first cell: $>1 \mathrm{~cm}$; street canyon width $20 \mathrm{~cm}$ ) to resolve the global flow structures.

At the inlet the measured boundary layer profiles (see [29]) for the velocity and TKE were imposed. Note that the boundary layer profiles were not measured at the beginning of the wind tunnel test section, but at the position of the centre of the street canyon, before the street canyon was installed. For the CFD simulations it was assumed that the boundary layers are fully developed and in equilibrium. Therefore these profiles can be used at the inlet of the computa- 
tional domain. This could lead to errors if the profiles further develop upstream of the street canyon in CFD, or the measured profiles were not fully developed (discussed below). The incoming air temperature was $23{ }^{\circ} \mathrm{C}$, to match the air temperature measured at the inlet of the test section. All surfaces were modelled without roughness, because no roughness can be defined with LRNM. This may be justified since the wind tunnel model was made out of aluminium with a very low surface roughness. For the surfaces inside the street canyon, the surface temperatures obtained from the experiments were used as a temperature boundary conditions and it is assumed that the surface temperatures were uniformly distributed over the surfaces. The surfaces outside the street canyon were modelled as adiabatic. Symmetry boundary conditions were imposed at the top boundary and outflow boundary conditions at the outlet.

To account for buoyancy, the density, the specific heat capacity, the thermal conductivity and the viscosity were approximated with polynomial functions as a function of the temperature in the Navier-Stokes equations. For all simulations the PRESTO! spatial discretization scheme was used for the pressure interpolation and second order spatial discretization schemes were used for the convection of the governing equations. Radiation was not considered directly in the CFD simulations, since constant temperature boundary conditions were imposed on the different street canyon surfaces.

\section{Validation Methodology}

To validate the CFD models with wind tunnel measurements, velocity and turbulent kinetic energy (TKE) profiles are compared. For some cases also contourplots and profiles of the temperature are used for validation. For the wind tunnel measurements the trajectories of the centre of the main vortices were introduced as criteria to characterise buoyant flow fields in urban street canyons [29]. Here these criteria are used to validate the CFD models. These criteria have advantages for cases where the main vortex is not in the centre of the street canyon due to buoyancy. It is not possible to use the standard approach of comparing profiles for cases where the centre of the main vortex in CFD and wind tunnel are not in the same position.

\section{Results}

\subsection{Approach flow}

In order to achieve a good agreement between CFD and measured flow fields inside the street canyon, the approach flow in CFD has to match the measured approach flow profiles. In Figure 3 the approach flow profiles of the wind tunnel measurements and the CFD simulations at $\mathrm{x}=7 \mathrm{~m}(0.4 \mathrm{~m}$ upstream of the street canyon) are given for the case with a Reynolds number of 19200 using two turbulence models (standard and realisable k- $\varepsilon$ model) and three near-wall treatments (LRNM, standard and non-equilibrium WFs). We find that in general the results are very similar for the two turbulence models. First the simulations were conducted employing LRNM. With LRNM the boundary layer is resolved. For both turbulence models there was a good agreement of the velocity profile using LRNM compared with the measured data. The TKEs were however too low for both turbulence models. The TKE was decaying in the streamwise direction in the CFD simulations. To avoid this decay shear stresses at the surfaces up- and down-stream of the street canyon were predefined together with TKE sources in the flow up- and down-stream of the street canyon (dashed lines "LRNM source"). With this method the streamwise change of the approach flow profiles of the TKE can be decreased. The higher TKEs had however an impact on the velocity profiles. The velocities in the near-wall regions decreased and became too low, while in the freestream they were too high.

Secondly, simulations were conducted using WFs. For the fine mesh used in this study, the first grid points were in the viscous sublayer. The flow quantities at the first grid point can be modelled with the law-of-the-wall used in the WFs. In contrast to the LRNM, the damping of TKE in the viscous sublayer and the buffer layer cannot be captured. This can lead to errors in the prediction of the TKE especially in the boundary layer. Due to this it is not recommended to use WFs for fine meshes. In this study WFs are nevertheless used, since the aim was to investigate if the results in the street canyon can be improved using WFs. Two types of WFs were used for the validation: the standard wall functions (SWFs) and the non-equilibrium wall functions (NEWFs) [35]. The NEWFs can partly account for the non-equilibrium 
effects that are neglected in the SWFs. Therefore the NEWFs can improve the results for complex flows involving separation, reattachment and impingement. Using the SWFs, the results for the velocity profiles deviated slightly more from measured values than for LRNM. The TKEs were slightly higher but still much lower compared to the measurements. As with LRNM, with source terms for the TKE the NEWFs underestimated the velocities in the near-wall regions and overestimated the velocities in the freestream. With NEWFs the peak of the TKE close to the ground can be captured, but the boundary layer was thicker than in the measurements.

Studying the approach flow profiles, it can be concluded that with the models employed in this study it is not possible to adjust both velocity and TKE profiles of the approach flow such that they match completely the measured profiles. For all simulations either the velocities or the TKEs in the near-wall regions are underestimated. The standard and realizable $\mathrm{k}-\varepsilon$ models give similar results for the approach flow.

\subsection{Isothermal case}

For the isothermal cases the energy equation is not solved. Therefore constant values for the air properties $\left(a t 15^{\circ} \mathrm{C}\right)$ are used instead of approximations as a function of the temperature. In Figure 4 the normalised horizontal centreline profiles of the velocity and TKE are given for the two turbulence models and three near-wall treatments for the case with a Reynolds number of 19200 . The velocity profiles show that all models predict the vortex in the centre of the street canyon. The results of the two turbulence models used gave very similar velocity profiles. The standard $k-\varepsilon$ model predicts slightly higher TKEs inside the street canyon than the realizable k- $\varepsilon$ model. Because the differences in TKEs do not cause a significant difference for the velocity profiles, it can be concluded that there is no strong coupling between the velocity and the TKE. The near-wall treatment has a large impact on the velocity and TKE profiles inside the street canyon. With LRNM the velocities are overestimated especially close to the walls. The results can be improved by using WFs (SWFs or NEWFs) or LRNM with a source term for the TKE in the freestream. In this case the results agree with the measured profiles. The CFD results differ more from the measured profiles for the TKEs than for the velocities. With the realizable k- $\varepsilon$ model the TKEs are too low in the centre of the street canyon for all approaches. For the WFs the TKEs are much too low in the centre of the street canyon. With LRNM a good match can be observed close to the windward wall, but the TKEs are overestimated close to the leeward wall. With the realizable k- $\varepsilon$ model the shapes of the TKE profiles are more similar to the measured profiles than with the standard k- $\varepsilon$ model. The best agreement can be found with the NEWFs. Thus for the isothermal street canyon case with a Reynolds number of 19200 the best results can be found for the NEWFs with a standard k- $\varepsilon$ model. Theoretically the LRNM should perform better, because it resolves the boundary layers and accounts for the damping of the TKE in the near-wall regions. Because the mesh is too fine at the walls for WFs, the NEWFs cannot account accurately for this damping of the TKE and therefore are expected to perform worse. However it can be observed that flow field results using NEWFs are the most accurate. There are several possible reasons for this. In the following section the influence of the approach flow profiles on the flow inside the street canyon is studied in more detail for the cases where all the surfaces inside the street canyon are heated. It will be shown that the velocity in the shear layer at the top of the street canyon has a strong influence on the flow inside the street canyon and that this may be the reason why the WFs perform better for the cases studied here.

\subsection{All surfaces heated}

In this section cases with all three street canyon surfaces heated to the same temperature are studied, using Froude numbers of (i) 6.75 (ii) 17.29 and (iii) 7.59. These three cases were chosen because CFD simulations and measurements show one main vortex in the centre of the street canyon, meaning that not only contour plots, but also centreline profiles can be compared.

In Figure 5 normalised centreline profiles of the horizontal velocity and TKE are given for the three measured cases of different Fr number and the corresponding CFD simulations. Here the profiles on the horizontal centerlines are given not only inside the street canyon as for the isothermal case, but also up to $0.75 \mathrm{H}$ above the street canyon. This allows studying the influence of the approach flow on the flow inside the street canyon. The following two CFD model combi- 
nations are used for the validation: (i) LRNM with a realizable $k-\varepsilon$ and (ii) NEWFs with a standard $k-\varepsilon$. The first was chosen because it is common combination of numerical models found often in literature. The second was chosen because it performs best for the isothermal case. Other simulations were conducted with the same models as the simulation with NEWFs, but the extension of the upstream domain in front of the street canyon was only $0.25 \mathrm{H}$ (NEWF short) instead of long extension of $37 \mathrm{H}$. With this short upstream domain, the boundary layer cannot develop and therefore the approach flow velocities do not decay as in the case with the long upstream domain. In this case the flow velocities at the entrance of the street canyon will correspond better to the measured values. The results for the velocities in Figure 5 show similar profiles for all three cases. In the boundary layer above the street canyon the simulation with NEWFs predicts velocities which are too low. With LRNM and NEWFs with the short domain, a very good agreement between the simulations and the measurements was found. In the shear layer at the top plane of the street canyon, the velocities are strongly decreasing and at $\mathrm{Y} / \mathrm{H}=1$ the slope of the profiles changes steeply. At this point the best agreement for the velocity can be found for the NEWFs, while the other two models overestimate the velocities. Inside the street canyon the best agreement can be found for simulations using NEWFs, while the other two models overestimate the velocities close to the ground. The results with LRNM and NEWFs with the short domain show very similar results. Therefore it can be concluded that velocities inside the street canyon are mainly influenced by the velocities in the shear layer. Even with velocities that are too low above the street canyon (using NEWFs) the flow inside the street canyon can be predicted well. For the other two models the results for the velocities are worse, even though there is a better agreement for the velocities above the street canyon. For the TKE all CFD models can capture the general trend. Above the street canyon the simulations with NEWFs overestimate the TKE and the simulations with LRNM underestimate the TKE. It can be observed that in the shear layer the two models with the long domain predict similar peak values. This is surprising, because the peak in TKE in the approach flow is significantly higher for the NEWFs compared to the LRNM (Figure 3). The peaks in the shear layer are lower for both models compared to the measurements for all three cases. The simulations with the short domain overestimate the peak in TKE inside the shear layer. Inside the street canyon the NEWFs (long and short domain) perform better than LRNM, which underestimates the TKE inside the street canyon. All CFD models predict the second peak close to the ground of the street canyon.

From the profiles in Figure 5 it can be concluded that it is important to correctly predict the flow in the approach flow as well as in the shear layer in order to get accurate results inside the street canyon. With the CFD models applied in this study the measured profiles in the shear layer cannot be satisfactorily captured. The most accurate results inside the street canyon can be found for the simulations with NEWFs, where the velocities inside the boundary layer are underestimated, but the velocity at building height $(\mathrm{Y} / \mathrm{H}=1)$ is correctly predicted. It was found that the simulated profiles in the shear layer cannot be improved with a mesh that is refined in this region. Therefore, it can be concluded that the overestimation of the velocities in the shear layer for the LRNM simulations is not due to a coarse grid. The flow structure in the shear layer seems to have a stronger impact on the flow field inside the street canyon than the near-wall treatment. This means that the error induced by not accounting for the damping of the TKE in the near-wall regions with NEWFs leads to a smaller error for the general flow structure compared the error induced by the inaccurate velocity predictions of the LRNM in the shear layer.

The shear layer is also important for the exchange of air between the street canyon and the flow above the street canyon, and therefore influences the temperature distribution inside the street canyon. In Figure 6 the contour plots of the air temperatures inside the street canyon are given for the three measured cases. Results of the measurements are compared with CFD simulations using LRNM and NEWFs. It can be observed that the LRNM simulations underestimate the air temperatures inside the street canyon with almost $10{ }^{\circ} \mathrm{C}$. With NEWFs the air temperatures in the centre of the street canyon are similar to the measured temperatures, but the thickness of the temperature boundary layers is overestimated. In Figure 7 horizontal profiles (measured, LRNM and NEWFs) of the temperature are given for the case with a Froude number of 6.75. It is expected that the most accurate results would be achieved by LRNM, where the boundary layer at the walls can be more accurately resolved. However with LRNM the temperatures are underestimated for all profiles by about $10^{\circ} \mathrm{C}$. The results can be improved by using NEWFs. For the profiles at $\mathrm{y}<0.05 \mathrm{~m}$ and for most profiles close to 
the windward and leeward walls the temperatures are overestimated by NEWFs due to the overestimated thickness of the temperature boundary layer. In the centre of the street canyon a good agreement for the temperatures can be found with NEWFs. A reason for the better agreement of the profiles with NEWFs compared to LRNM could be that the velocities in the shear layer correspond better to the measured velocities (see Figure 5) and therefore the predicted air exchange rate is more accurate. Another possible reason is that the convective heat transfer predictions are more accurate due to the different near-wall treatment. This cannot be evaluated here, because the wall heat fluxes were not measured by Allegrini et al. [29].

\subsection{Windward wall heating}

For windward wall heating the vortex structure is strongly influenced by buoyancy. Streamlines for five different Reynolds numbers and a windward wall temperature of $130^{\circ} \mathrm{C}$ are presented in Figure 8 [29]. Besides the main vortex a second counter rotating vortex is formed. The size and location of the main and secondary vortex are changing with increasing Reynolds number. Therefore the information gained from comparing centreline profiles is rather limited, because small changes in the location of the centre of the vortices strongly change the profiles. As in Allegrini et al. [29] the trajectories of the centre of the main vortex are studied in this section. Figure 9 shows the trajectories for the measured flow fields and the results of simulations with NEWF and LRNM. The (measured) results indicated with $23{ }^{\circ} \mathrm{C}$ show the locations of the centre of the main vortex for the isothermal cases. For the isothermal cases the vortex centres are close to the centre of the street canyon for all Reynolds numbers. The location of the main vortex changes only slightly for increasing Reynolds numbers. For the measurements with windward wall temperatures of $70{ }^{\circ} \mathrm{C}$ and $130{ }^{\circ} \mathrm{C}$ the centre of the main vortex moved from the leeward wall side towards the windward wall side and then to the centre of the street canyon with increasing freestream velocities. The trajectories are very similar for both windward wall temperatures. The strength of buoyancy is smaller for windward wall temperatures of $70^{\circ} \mathrm{C}$ and therefore for high freestream velocities the centre of the main vortex is closer to the isothermal cases than for windward wall temperatures of $130{ }^{\circ} \mathrm{C}$. The trajectories for the results from CFD are close together. Although there is a difference between the trajectories from the measurement and CFD, the CFD results show the same tendencies. Also with CFD the vortex centres moves from the leeward wall side towards the windward wall side and then to the centre of the street canyon with increasing freestream velocities. The CFD simulations are not sufficiently accurate to predict the detailed flow field for each case, but they can predict the general vortex structure. The trajectories are similar for the simulations with LRNM and NEWFs.

\subsection{Leeward wall heating}

For the cases with leeward wall heating the validation is conducted for a Reynolds number of 19200 with different surface temperatures. The CFD results for the normalised horizontal velocities and the TKEs on the vertical centreline obtained using LRNM and NEWFs are compared with the wind tunnel measurements (Figures 10 and 11). As for the cases where all surfaces are heated, the velocities at the top of the street canyon are overestimated with LRNM. Therefore the velocities at the ground of the street canyon are also overestimated. The velocity profiles for the simulations using NEWFs match the measured profiles well. Also for the TKE the results of the simulations using NEWFs show a better agreement with the measured results than the simulations using LRNM.

\section{Discussion}

\subsection{Model limitations}

This validation study focuses on buoyant flows in a square cavity representing an urban street canyon. Buoyant flow fields have commonly unsteady and 3D flow characteristics. The unsteadiness and the three-dimensionality of the flow cannot be captured with the 2D steady RANS simulations that are conducted for this validation study. We conducted this study under the assumption that the flow within the studied plane is in a mixed or forced flow regime, where still a high degree of flow two-dimensionality can be found. For natural convection, where the flow is strongly threedimensional, 3D simulations would be needed. The strongly simplified CFD models were chosen because they are ap- 
plicable for full-scale flows in street canyons and were already commonly used in literature, but to the authors' knowledge never validated in detail. The aim of this validation study was not to find the most accurate CFD models to predict the detailed flow field inside a heated street canyon, but to study the extent to which the most important flow phenomena can be captured with these simplified CFD models (steady and 2D simulations). It is assumed that the most accurate results can be found for time resolved 3D CFD simulations (e.g. LES: large eddy simulation). However, with today's available computational power, it is for most cases not feasible to apply these more advanced CFD models for full-scale building applications.

\subsection{Interaction between flow field and convective heat flux}

Due to buoyancy there is a strong coupling between the flow field inside the street canyon and the heat fluxes at the boundaries of the street canyon. Therefore small errors in the prediction of the fluxes or the flow field can significantly change the results of the CFD simulations. In the case of the street canyon the important fluxes are the convective heat fluxes at the three surfaces of the street canyon, the heat flux out of the street canyon into the freestream and the heat flux into the street canyon from the freestream (red arrows in Figure 12). The temperature distribution inside the street canyon is strongly dependent on the balance between these heat fluxes.

The main driver of all the fluxes and the resulting temperature distribution is the heat exchange at the top plane of the street canyon. The area for this heat exchange is indicated with the blue box in Figure 12 and the heat exchange is sketched with the blue arrows. The upstream air is mixed with the air leaving the street canyon. A part of the mixed air is entering / re-entering the street canyon and another part is transported further downstream of the street canyon (blue arrows). Theoretically there exist two extreme cases. For the first there is no heat exchange at the top plane of the street canyon. As soon as the fluxes are in equilibrium, the air temperature inside the street canyon is the same as the temperature at the (uniformly heated) surfaces and there is no convective heat transfer at the surfaces. Due to the uniform temperature distribution, buoyancy does not influence the flow and a forced convective flow can be found inside the street canyon. The second extreme case is the case with an infinite heat exchange at the top plane, so that all heat added to the flow by convection inside the street canyon is removed instantly. Therefore the equilibrium air temperature inside the street canyon is the temperature of the freestream above the street canyon. Also in this extreme case a uniform temperature distribution inside the street canyon can be found and buoyancy does not influence the (forced convective) flow.

In reality the heat exchange at the top plane of the street canyon is somewhere between these two extreme values, the temperatures are between the two extremes and the temperature distribution inside the street canyon is not uniform. For a case with one main vortex in the centre of the street canyon, the temperature at the windward wall, where the cold air of the freestream is entering the street canyon, is lower compared to the temperature at the leeward wall, where a part of the heated air is leaving the street canyon. Due to this non-uniform temperature distribution, buoyancy starts to play an important role and the flow structure becomes dependent on the temperature distribution.

The heat exchange at the top plane is driven by the flow structure at the top plane, because it is caused by the air exchange. The air exchange is driven by a combination of convection and turbulence. On the other hand the flow structure is strongly influenced by the heat exchange at the top plane, because this exchange has an impact on the temperature distribution and therefore on the buoyancy effects. This explains the strong coupling between the heat fluxes and the flow structures inside a street canyon. For example an increase of the leeward wall temperature can increase the heat exchange at the top plane due to increased buoyancy effects. This increased heat exchange can lead to lower air temperatures at the windward wall and a significant change in the whole flow structure inside the street canyon. This change in the flow structure has an impact on all the heat fluxes and due to the coupling the flow structure will change further. This leads to the conclusion that for a validation of the flow inside a street canyon, in addition to the flow characteristics the heat fluxes also need to be studied. Only if the flow structures and the heat fluxes are analysed a complete validation of the important physical phenomena can be conducted. The focus of such a validation study should be on the heat exchange at the top plane of the street canyon, because (as explained above) this heat exchange drives all physical phenomena inside the street canyon. 
The heat exchange at the top plane is strongly connected to the air exchange at the top plane. Analysing only the vertical velocities at the top plane is not sufficient to find the air exchange rate of the street canyon. Air leaving the street canyon at one position at the top plane could reenter at a different position, and air entering the street canyon could leave again without being mixed with the air inside. For example if there is one main vortex in the centre of the street canyon and the diameter of this vortex is larger than the height of the street canyon, most of the air leaving the street canyon at the leeward wall will reenter at the windward wall again. Alternatively, if the diameter of the vortex is smaller than the height of the street canyon, most of the air entering the street canyon at the leeward wall may leave again at the windward wall (Figure 13). Further above the top plane of the street canyon the air in the approach flow mixes with the air leaving the street canyon, and therefore the temperature of the air entering the street canyon does not correspond to the approach flow air temperature for most cases. Therefore for most cases no surface can be defined where the heat exchange can be studied by analysing only the wind velocities. This leads to the conclusion that the heat fluxes themselves have to be analysed.

For this validation study only the flow fields were measured in detail. To be able to determine heat fluxes the detailed temperature fields also need to be known with a high resolution. In the paper of Allegrini et al. [29] the temperature fields were measured for a few cases, but at too a low resolution to determine heat fluxes. To determine the heat balance of the street canyon the convective heat fluxes at the surfaces of the street canyon also have to be measured. In addition to the flow field inside the street canyon, the flow field above the street canyon and especially the flow field in the shear layer must also be known for the validation. As discussed here, this region is important for the heat exchange at the top plane and it is also important for the velocity prediction with CFD as discussed in the sections above. Therefore it is suggested that additional wind tunnel measurements be conducted, focusing on the region indicated with the blue box in Figure 12. This volume contains the important transport phenomena that drive the ventilation of the street canyon. Determining a heat balance for this control volume allows detailed study of the heat fluxes, which not only influence the heat exchange at the surfaces of the street canyon but also the flow field.

\subsection{Computational mesh}

In this study the same mesh was used for LRNM and WFs. The mesh was optimised for LRNM and was therefore too fine at the surfaces for WFs, because the first grid point was inside the viscous sublayer. Therefore for the simulations with WFs the damping of the TKE in the boundary layer was not considered. This leads to an error in the simulated flow fields. The results in this study show that the error introduced in the flow inside the street canyon by using an inappropriate mesh is smaller than the error introduced due to the wrong prediction of the velocities at the top plane of the street canyon with LRNM. This can be seen in Figure 5, where the profiles for simulations using NEWFs and LRNM give similar profiles for the velocity, if the velocity profile at the top plane of the street canyon and above the street canyon are almost the same. That means that not accounting for the damping of TKE in the boundary layer does not significantly change the flow inside the street canyon. The most accurate results are therefore expected for CFD simulations with LRNM, where the shear layer can be accurately modelled.

\section{Conclusion}

A validation study was conducted for buoyant flows in street canyons. For the validation the CFD results were compared with our own wind tunnel measurements. 2D RANS simulations were conducted with k- $\varepsilon$ turbulence models and different near-wall treatments. Low-Reynolds number modelling (LRNM) and wall functions (WFs) were applied on the same mesh that was optimized for LRNM. When using WFs on that mesh in the near-wall region, damping of turbulent kinetic energy (TKE) cannot be accounted for. Therefore an error is introduced by using WFs on a mesh optimized for LRNM.

It was found that the general flow structures can be captured with all the CFD models used. Differences between the different CFD models and between CFD and measurement results can be observed when comparing detailed flow profiles. In the approach flow the profiles of the velocity and TKE cannot be captured correctly in the same simulation. Ei- 
ther the velocity profile is correct but with a too strong a streamwise decay of the TKE (using LRNM), or the TKE profiles are correct but with the velocities in the near-wall regions decreasing in the streamwise direction (using nonequilibrium wall functions: NEWFs). Using LRNM the velocities in the near-wall regions are overestimated for all cases studied, while with NEWFs a significantly better agreement can be observed. A possible reason for the better agreement are the lower (too low compared to the measurements) velocities in the boundary layer above the street canyon. Due to this the velocities at the top plane of the street canyon agree better with the measured velocities, while with LRNM these velocities are overestimated. This overestimation in the top plane could cause the overall overestimation of the velocities inside the street canyon. This is to a certain extent confirmed by simulations with NEWFs using a very short upstream domain, where the boundary layer cannot develop in front of the street canyon. For this case the velocity profile of the approach flow is the same for LRNM and NEWFs and also the velocities inside the street canyon are comparable. These results lead to the conclusion that the error introduced by not considering the damping of TKE in the near-wall region with NEWFs is lower than the error introduced by the overestimation of the velocity at the top plane of the street canyon with LRNM. Similar shapes of the TKE profiles can be observed for the measurements and the CFD simulations using NEWFs. The standard k- $\varepsilon$ turbulence model predicts higher TKEs than the realizable k- $\varepsilon$ model. The best results for the TKE can mostly be found with the realizable k- $\varepsilon$ if LRNM is applied and with the standard k- $\varepsilon$ when NEWFs are used. Inside the street canyon the turbulence model has no strong influence on the velocities.

For the buoyant cases the use of NEWFs gives better results for the trajectories of the main vortex than using LRNM. Simulations using LRNM underestimate the temperatures largely inside the street canyon, while a good agreement can be observed with NEWFs in the centre of the street canyon. However, NEWFs overestimate the thickness of the temperature boundary layer. The better agreement for temperatures could be caused by the better agreement of the flow at the top plane of the street canyon, or due to differences in the modelling approach of the thermal boundary layer.

This study shows that CFD can be useful to predict flows in buoyant urban street canyons and that the general flow structure can be captured. However, to analyse the detailed flow structures and heat fluxes a validation study with more detailed temperature and flow measurements at the top plane of the street canyon needs to be conducted. To get the best possible agreement between CFD and measurements, more complex CFD models have to be used. These models should account for the unsteady and 3D characteristics of the buoyant flow field inside a street canyon.

\section{Acknowledgments}

Funding by the Swiss Federal Office of Energy (SFOE) is gratefully acknowledged (Project Number: 154 143, Project Title: Urban climate and energy demand in buildings).

\section{References}

[1] Moonen P, Defraeye T, Dorer V, Blocken B, Carmeliet J. Urban physics: Effect of the micro-climate on comfort, health and energy demand. Front Archit Res 2012;1(3): 197-228.

[2] Stathopoulos T. Pedestrian level winds and outdoor human comfort. J Wind Eng Ind Aerod 2006;94(11):769-80.

[3] Blocken B, Persoon J. Pedestrian wind comfort around a larger football stadium in an urban environment: CFD simulation, validation and application of the new Dutch wind nuisance standard. J Wind Eng Ind Aerod 2009;97(5-6):25570.

[4] Blocken B, Janssen W, van Hooff T. CFD simulation for pedestrian wind comfort and wind safety in urban areas: General decision framework and case study for the Eindhoven university campus. Environ Model Softw 2012;30:15-34.

[5] Gousseau P, Blocken B, Stathopoulos T, van Heijst G. CFD simulation of near-field pollutant dispersion on a highresolution grid: A case study by LES and RANS for a building group in downtown Montreal. Atmos Environ 2011;45(2):428-38. 
[6] Sabatino SD, Buccolieri R, Pulvirenti B, Britter R. Simulations of pollutant dispersion within idealised urban-type geometries with CFD and integral models. Atmos Environ 2007;41(37):8316-29.

[7] Tominaga Y, Stathopoulos T. CFD modeling of pollution dispersion in a street canyon: Comparison between LES and RANS. J Wind Eng Ind Aerod 2011;99(4):340-48.

[8] Moonen P, Gromke C, Dorer V. Performance assessment of Large Eddy Simulation (LES) for modeling dispersion in an urban street canyon with tree planting. Atmos Environ 2013;75:66-76.

[9] Bouyer J, Inard C, Musy M. Microclimatic coupling as a solution to improve building energy simulation in an urban context. Energy Build 2011;43(7):49-1559.

[10] Allegrini J, Dorer V, Carmeliet J. Analysis of convective heat transfer at building façades in street canyons and its influence on the space cooling demand in buildings. J Wind Eng Ind Aerod 2012;104-105:464-73.

[11] Van Hooff T, Blocken B. CFD evaluation of natural ventilation of indoor environments by the concentration decay method: $\mathrm{CO}_{2}$ gas dispersion from a semi-enclosed stadium. Build Environ 2013;61:1-17.

[12] Hertwig D, Efthimiou GC, Bartzis JG, Leitl B. CFD-RANS model validation of turbulent flow in a semi idealized urban canopy. J Wind Eng Ind Aerod 2012;111:61-72.

[13] Kubilay A, Derome D, Blocken B, Carmeliet J. CFD simulation and validation of wind-driven rain on a building façade with an Eulerian multiphase model. Build Environ 2013;61:69-81.

[14] Montazeri H, Blocken B. CFD simulation of wind-induced pressure coefficients on buildings with and without balconies: Validation and sensitivity analysis. Build Environ 2013;60:137-49.

[15] Kovar-Panskus A, Louka P, Sini JF, Savory E, Czech M, Abdelqari A, Mestayer PG, Toy N. Influence of geometry on the mean flow within urban street canyons - a comparison of wind tunnel experiments and numerical simulations. Water Air Soil Pollut 2002;2:365-80.

[16] Chan T, Dong G, Leung C, Cheung C, Hung W. Validation of a two-dimensional pollutant dispersion model in an isolated street canyon. Atmos Environ 2002;36(5):861-72.

[17] Meroney RN, Pavageau M, Rafailidis S, Schatzmann M. Study of line source characteristics for 2-D physical modeling of pollutant dispersion in street canyons. J Wind Eng Ind Aerod 1996;62(1):37-56.

[18] Memon RA, Leung DY, Liu CH. Effects of building aspect ratio and wind speed on air temperatures in urban like street canyons. Build and Environ 2010;45(1):176-88.

[19] Xie X, Liu CH, Leung DY, Leung MK. Characteristics of air exchange in a street canyon with ground heating. Atmos Environ 2006;40(33):6396-409.

[20] Kim JJ, Baik JJ. Urban street-canyon flows with bottom heating. Atmos Environ 2001;35(20):3395-404.

[21] Kim JJ, Baik JJ. Effects of street-bottom and building roof heating on flow in three-dimensional street canyons. Adv Atmos Sci 2010;27:513-27.

[22] Uehara K, Murakami S, Oikawa S, Wakamatsu S. Wind tunnel experiments on how thermal stratification affects flow in and above urban street canyons. Atmos Environ 2000;34(10):1553-62.

[23] Xie X, Liu CH, Leung DY, Impact of building facades and ground heating on wind flow and pollutant transport in street canyons. Atmos Environ 2007;41(39):9030-49.

[24] Kovar-Panskus A, Moulinneuf L, Savory E, Abdelqari A, Sini JF, Rosant JM, Robins A, Toy N. A wind tunnel investigation of the influence of solar induced wall-heating on the flow regime within a simulated urban street canyon. Water Air Soil Pollut 2002;2:555-71. 
[25] Tablada A, Roels S. Numerical study on the influence of wind and thermal stack on street canyon airflow pattern. Yokohama, Japan: The $7^{\text {th }}$ international conference on urban climate (ICUC 7); 2009.

[26] Garbero V, Salizzoni P, Marro M, Berrone S, Soulhac L. Influence of heat fluxes on the flow within a twodimensional street canyon: a comparison between wind tunnel measurements and CFD simulations. Amsterdam, Netherlands: $13^{\text {th }}$ International Conference on Wind Engineering; 2011.

[27] Dimitrova R, Sini JF, Richards K, Schatzmann M, Weeks M, Perez Garcia E, Borrego C. Influence of thermal effects on the wind field within the urban environment. Bound-Layer Meteorol 2009;131:223-43.

[28] Assimakopoulos V, Georgakis C, Santamouris M. Experimental validation of a computational fluid dynamics code to predict the wind speed in street canyons for passive cooling purposes. Sol Energy 2006;80:423-34.

[29] Allegrini J, Dorer V, Carmeliet J. Wind tunnel measurements of buoyant flows in street canyons. Build Environ 2013;59:315-26.

[30] Saneinejad S, Moonen P, Defraeye T, Derome D, Carmeliet J. Coupled CFD, radiation and porous media transport model for evaluating evaporative cooling in an urban environment. J Wind Eng Ind Aerod 2012;104-106:455-63.

[31] Huang Y, Hu X, Zeng N. Impact of wedge-shaped roofs on airflow and pollutant dispersion inside urban street canyons. Build Environ 2009;44(12):2335-47.

[32] Fluent 12.0 user's guide. USA: Lebanon - New Hamphire; 2009.

[33] Oberkampf WL, Trucano TG. Verification and validation in computational fluid dynamics. Prog Aerosp Sci 2002;38:209-72.

[35] Franke J, Hellsten A, Schlünzen H, Carissimo B. Best practice guideline for the CFD simulation of flows in the urban environment. COST Action 732: quality assurance and improvement of mesoscale meteorological models; 2007; Hamburg, Germany.

[35] Kim SE, Choudhury D. A near-wall treatment using wall functions sensitized to pressure gradient. ASME FED $217 ; 1995$. 


\section{Figures}

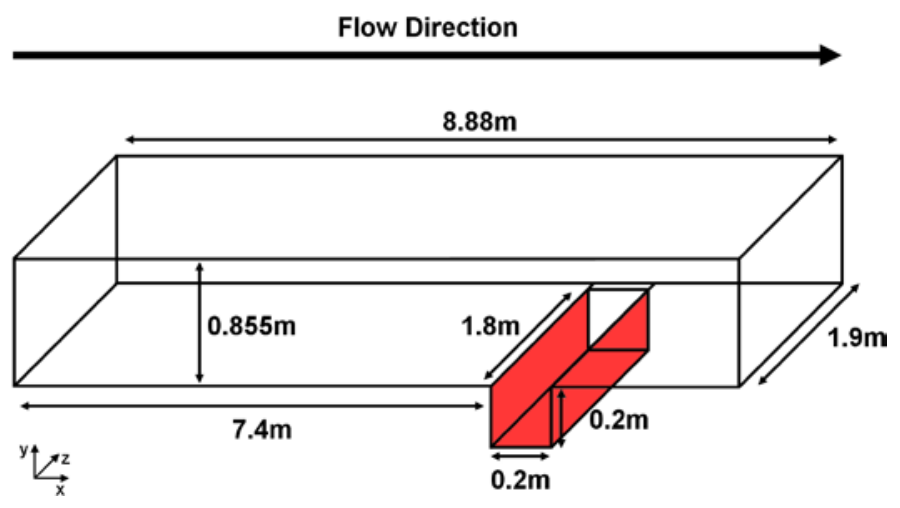

Figure 1: Dimensions of the wind tunnel model.

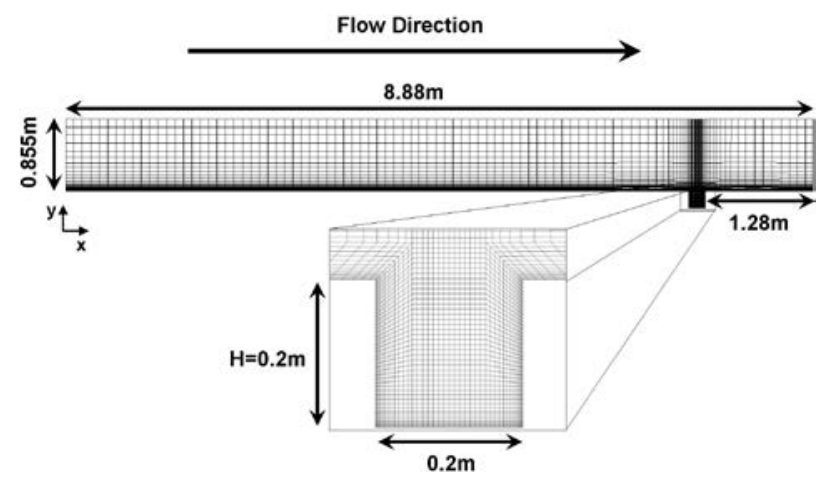

Figure 2: Computational domain.
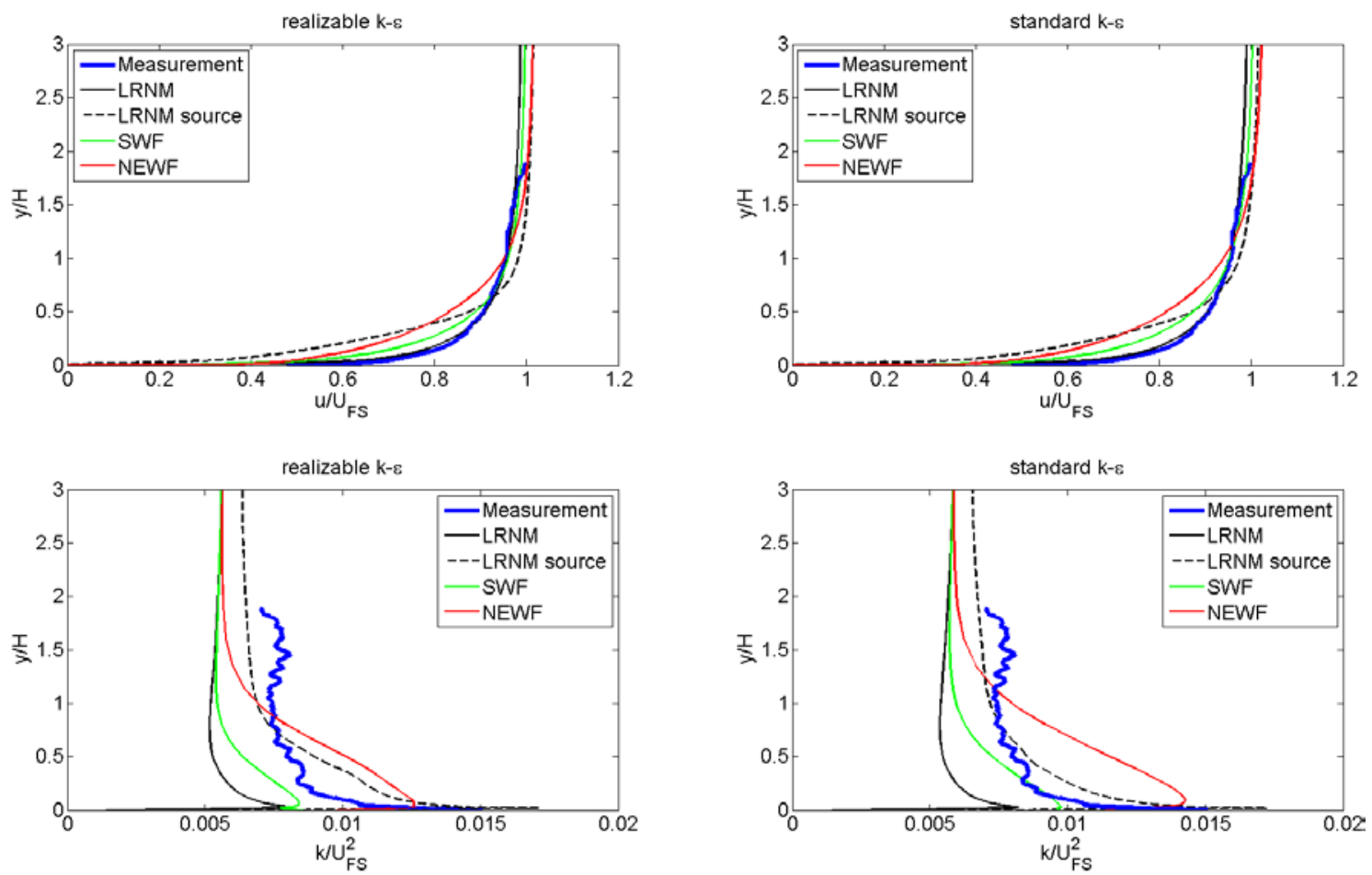

Figure 3: Normalised approach flow profiles of the velocity and TKE. Wind tunnel measurements and CFD simulations for the case with a Reynolds number of 19200 using two different turbulence models and different near-wall modelling approaches (LRNM: Low-Reynolds number modelling; LRNM source: LRNM with source terms for k and predefined shear stresses at the surfaces up- and down-stream of the street canyon; NEWF: non-equilibrium wall functions; SWF: standard wall functions). 

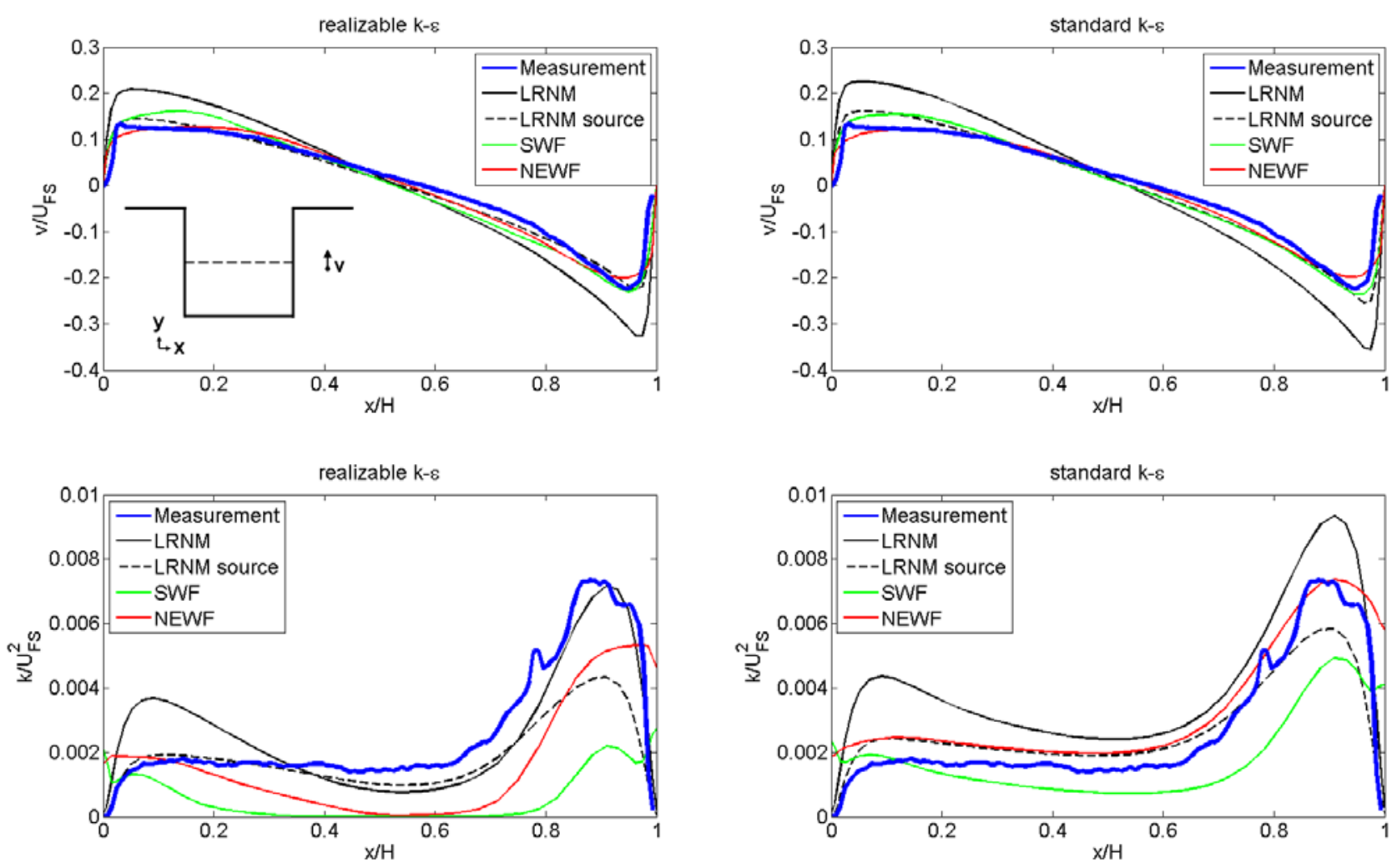

Figure 4: Normalised horizontal centreline profiles of the vertical velocity and TKE. Wind tunnel measurements and CFD simulations for the case with a Reynolds number of 19200 using different turbulence models and different nearwall modelling approaches (LRNM: Low-Reynolds number modelling; LRNM source: LRNM with source terms for $\mathrm{k}$ and predefined shear stresses at the surfaces up- and down-stream of the street canyon; NEWF: non-equilibrium wall functions; SWF: standard wall functions).
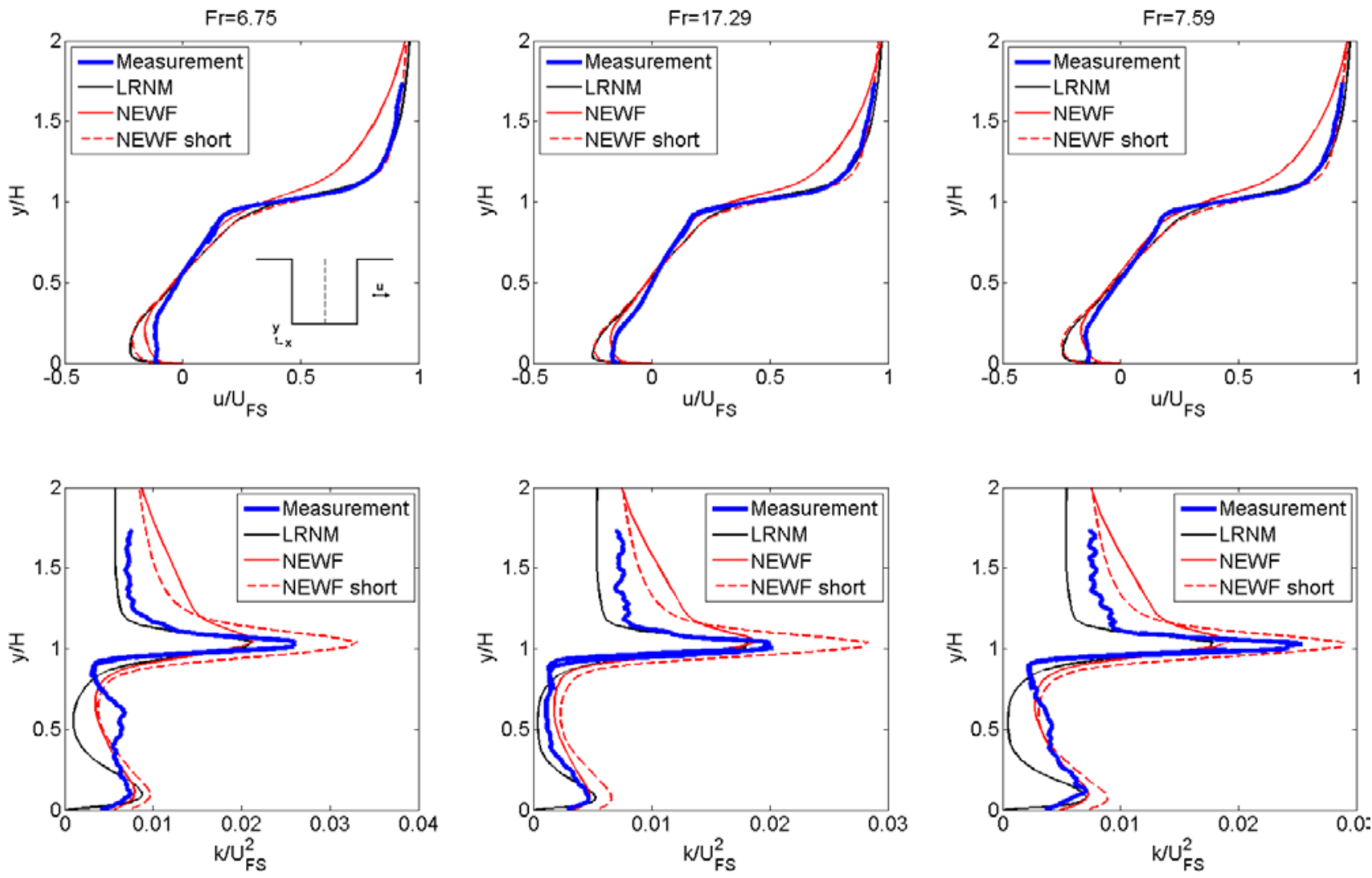
Figure 5: Normalised vertical centreline profiles of the horizontal velocity and TKE. Wind tunnel measurements and CFD simulations for cases where all surfaces are equally heated, using different turbulence models and different nearwall modelling approaches (LRNM: Low-Reynolds number modelling with realizable k- $\varepsilon$; NEWF: non-equilibrium wall functions with standard k-e; NEWF short: NEWF with a short upstream domain).
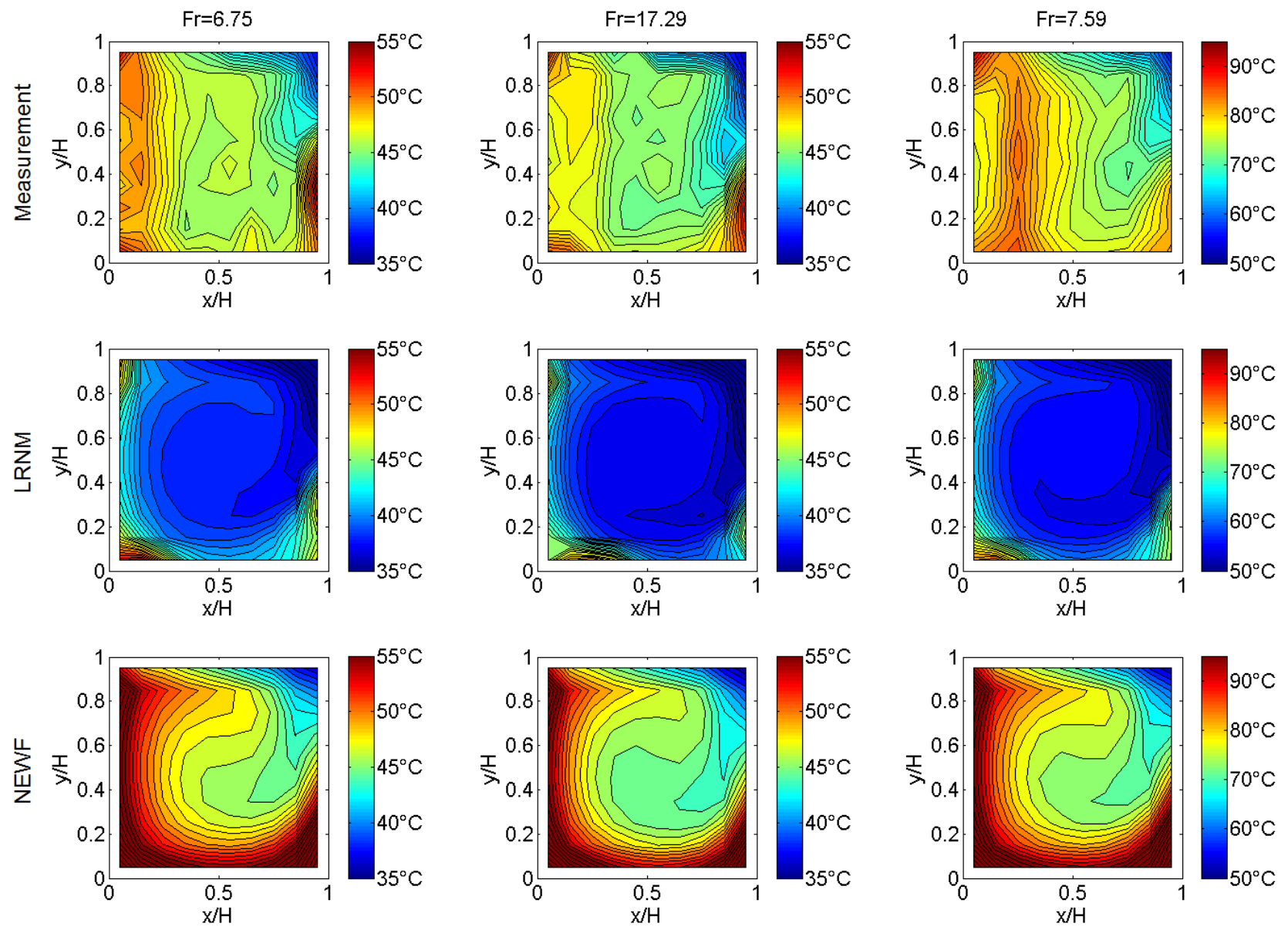

Figure 6: Contour plots of the air temperatures inside the street canyon. Wind tunnel measurements and CFD simulations for cases where all surfaces are equally heated using two different turbulence models and different near-wall modelling approaches (LRNM: Low-Reynolds number modelling with realizable k- $\varepsilon$; NEWF: non-equilibrium wall functions with standard k- $\varepsilon$ ). Note different temperature scales. $\mathrm{Fr}=6.75: \operatorname{Re}=19200$ and $\mathrm{T}_{\mathrm{W}}=70{ }^{\circ} \mathrm{C} ; \mathrm{Fr}=17.29: \operatorname{Re}=$ 30700 and $\mathrm{T}_{\mathrm{W}}=70^{\circ} \mathrm{C} ; \mathrm{Fr}=7.59: \mathrm{Re}=30700$ and $\mathrm{T}_{\mathrm{W}}=130^{\circ} \mathrm{C}$. 

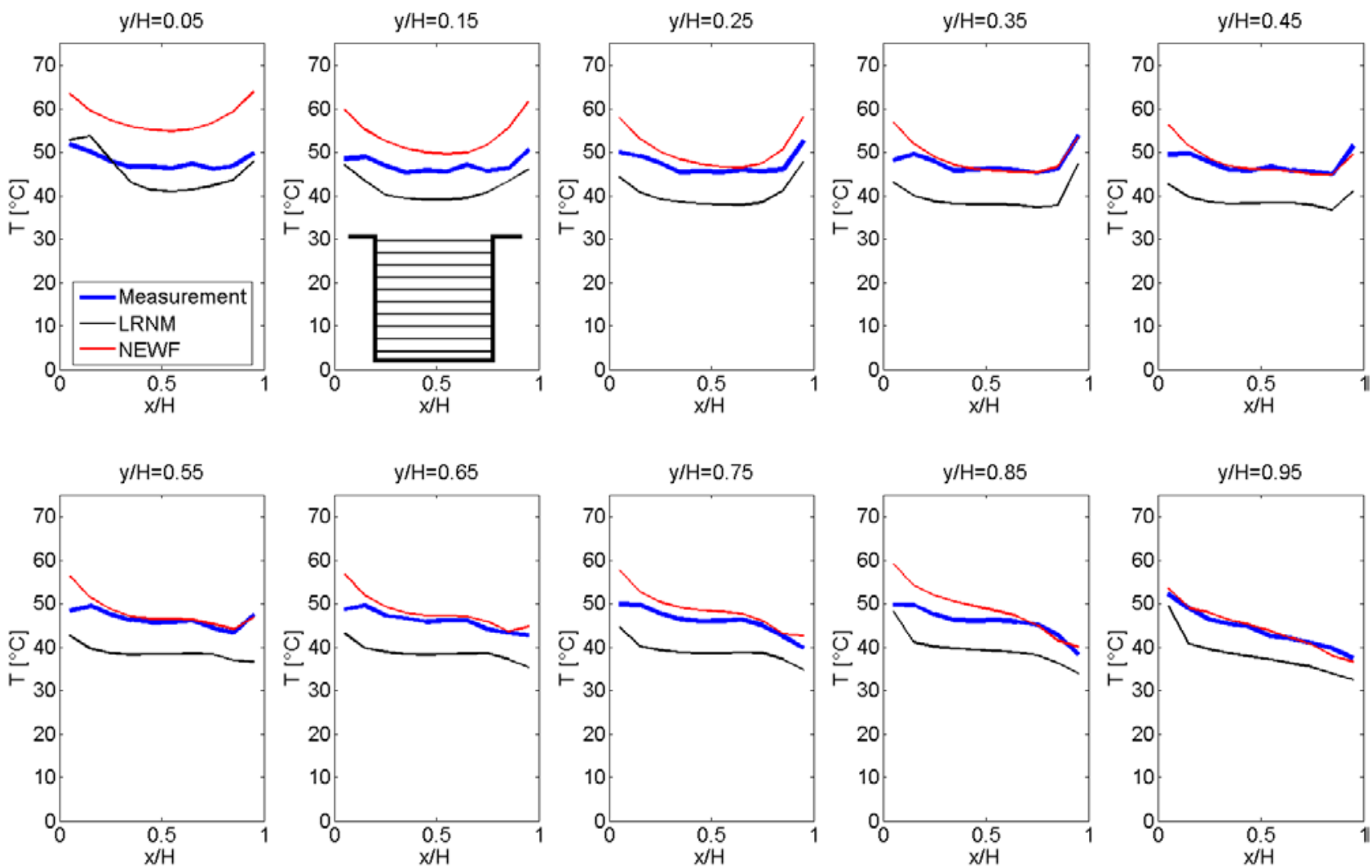

Figure 7: Temperature profiles on horizontal lines at different y-positions inside the street canyon. Wind tunnel measurements and CFD simulations for cases where all surfaces are heated $(\mathrm{Fr}=6.75)$ using two different turbulence models and different near-wall modelling approaches (LRNM: Low-Reynolds number modelling with realizable k- $\varepsilon$; NEWF: non-equilibrium wall functions with standard $k-\varepsilon)$.
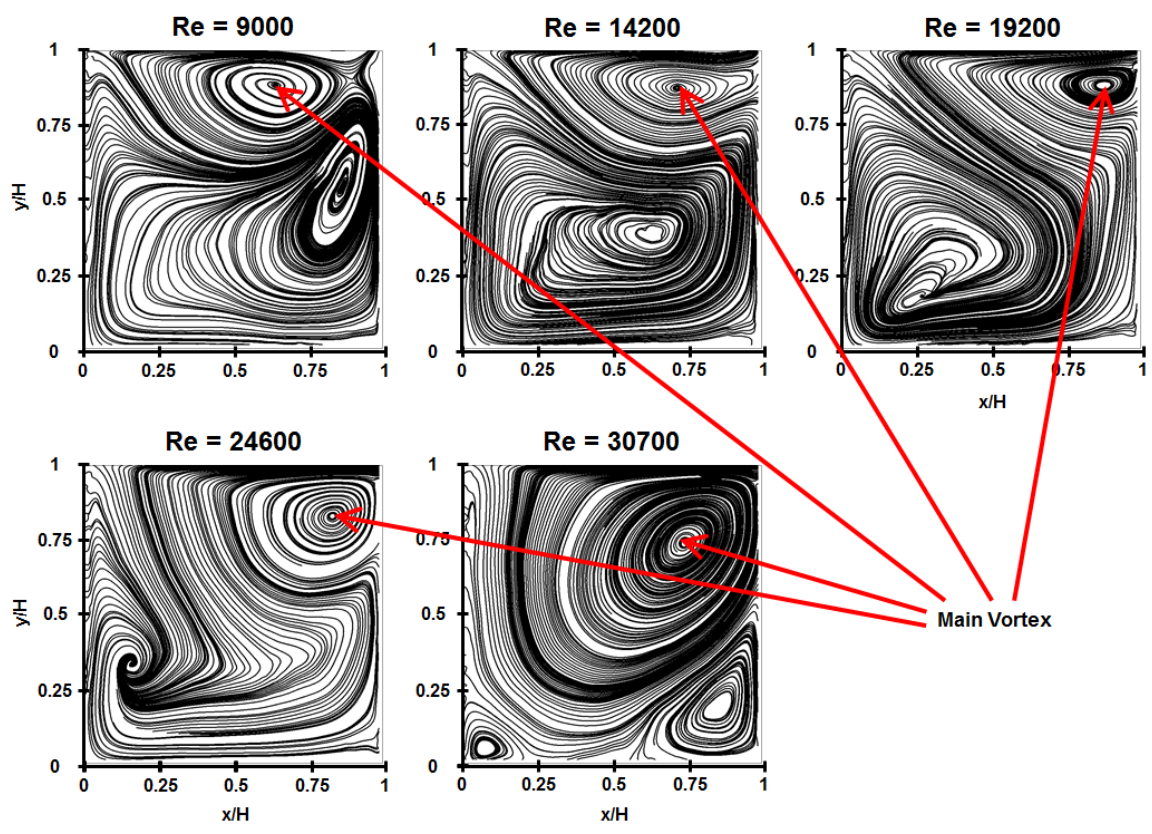

Figure 8: Streamlines for cases with windward wall heating and five different Reynolds numbers. 


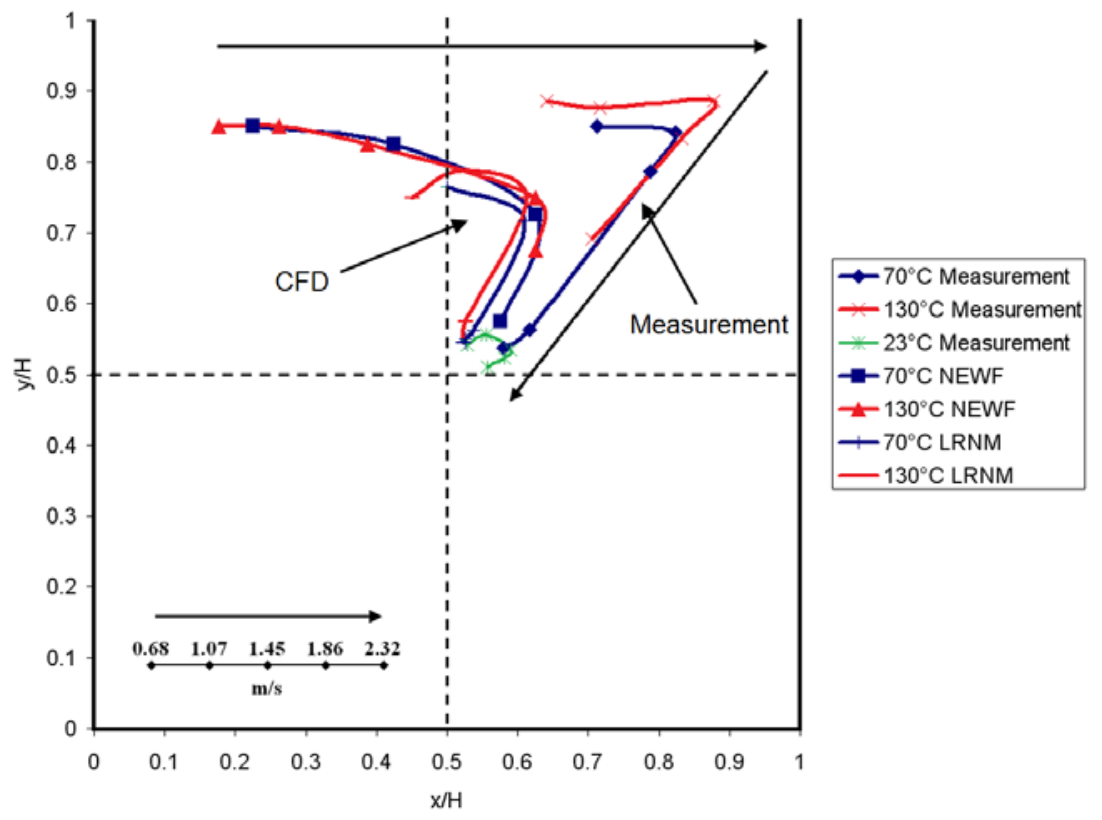

Figure 9: Trajectories of the centre of the main vortex as a function of the freestream velocity for different windward wall temperatures. Wind tunnel measurements and CFD simulations using two different turbulence models and different near-wall modelling approaches (LRNM: Low-Reynolds number modelling with realizable k- $\varepsilon$; NEWF: nonequilibrium wall functions with standard $\mathrm{k}-\varepsilon$ ).
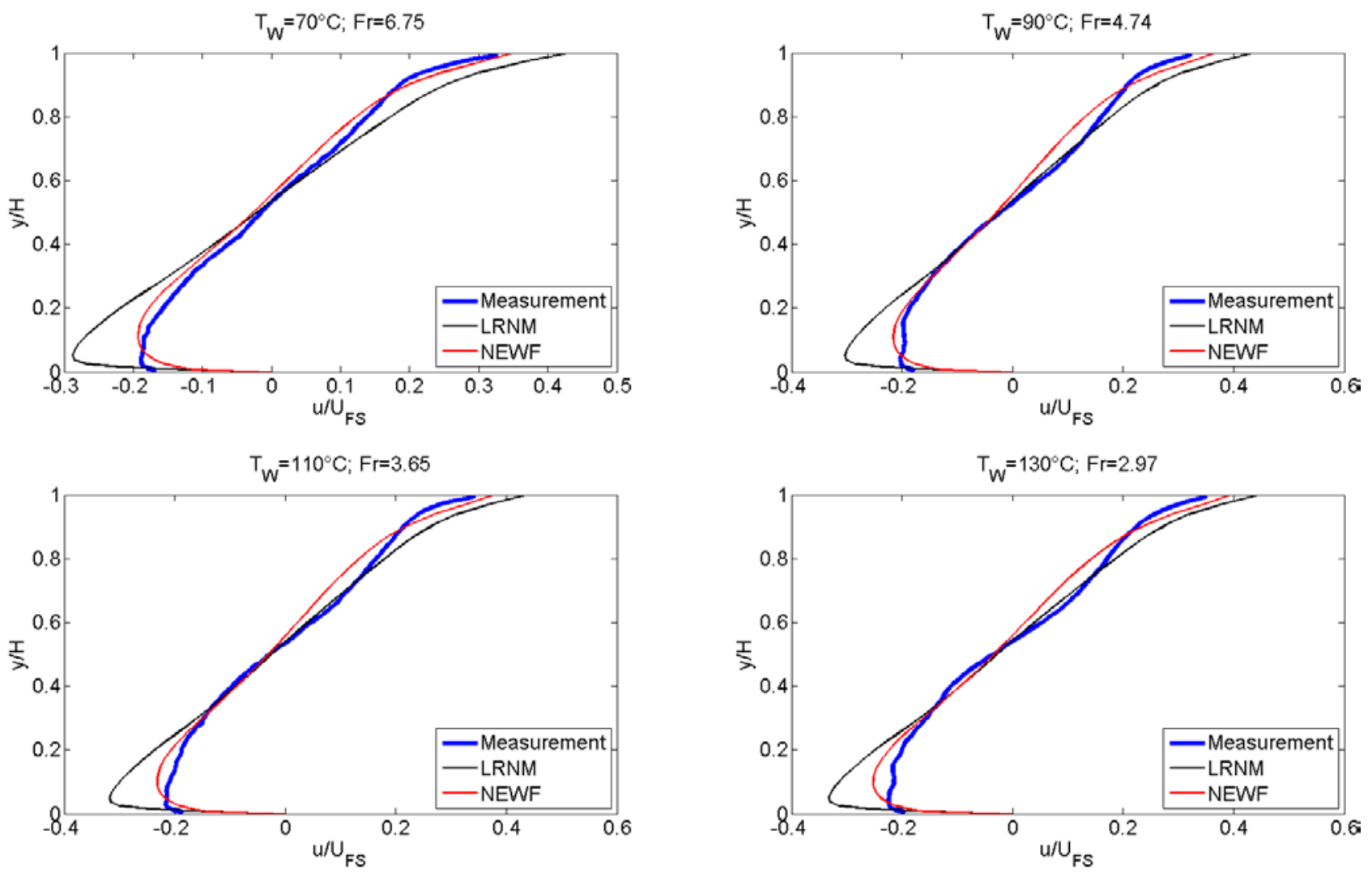

Figure 10: Normalised vertical centreline profiles of the horizontal velocity for different surface temperatures $(\mathrm{Re}=19200)$. Wind tunnel measurements and CFD simulations using two different turbulence models and different near-wall modelling approaches (LRNM: Low-Reynolds number modelling with realizable k- $\varepsilon$; NEWF: nonequilibrium wall functions with standard $\mathrm{k}-\varepsilon$ ). 

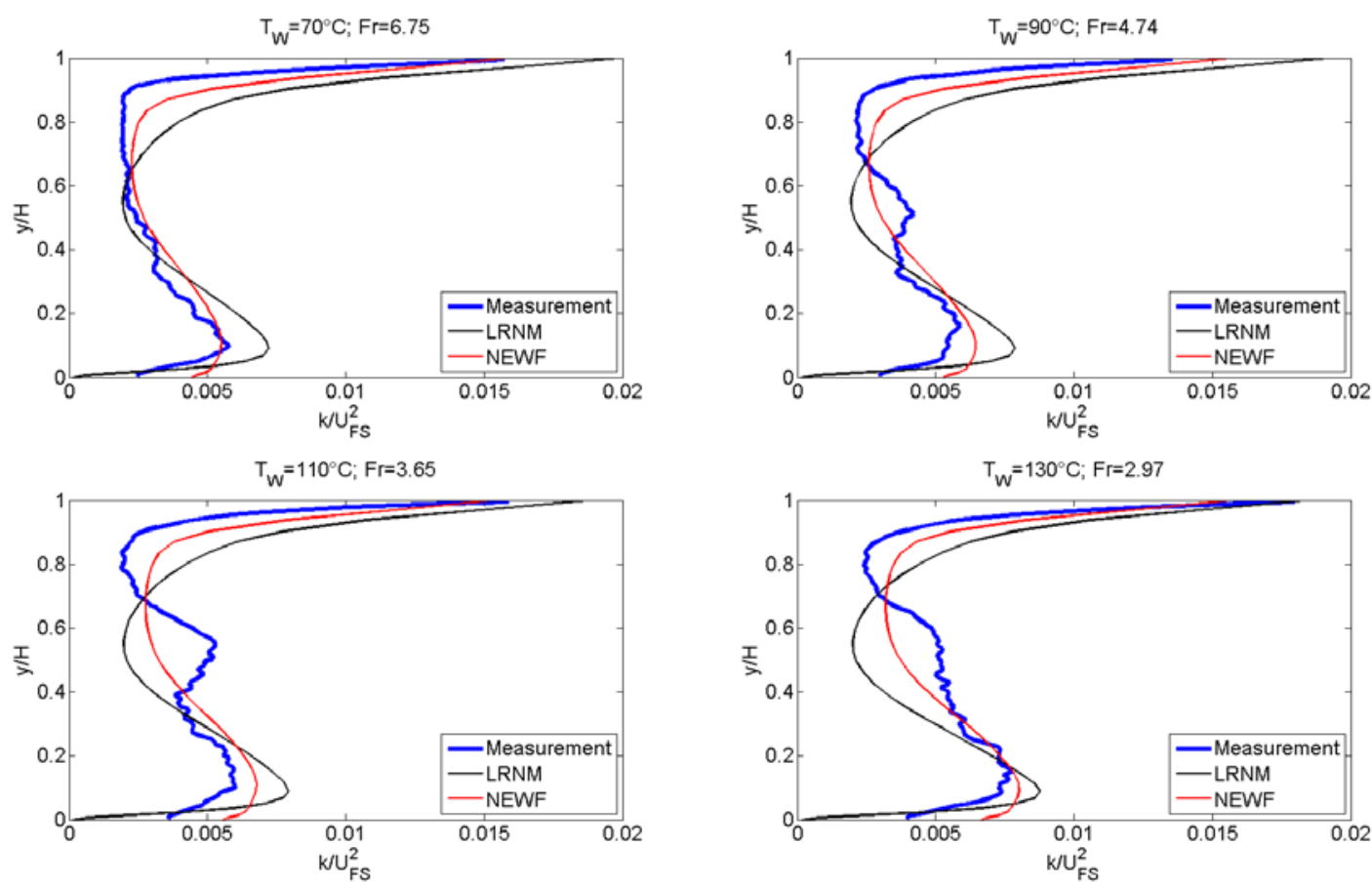

Figure 11: Normalised vertical centreline profiles of the TKE for different surface temperatures $(\operatorname{Re}=19200)$. Wind tunnel measurements and CFD simulations using two different turbulence models and different near-wall modelling approaches (LRNM: Low-Reynolds number modelling with realizable k- $\varepsilon$; NEWF: non-equilibrium wall functions with standard $\mathrm{k}-\varepsilon)$.

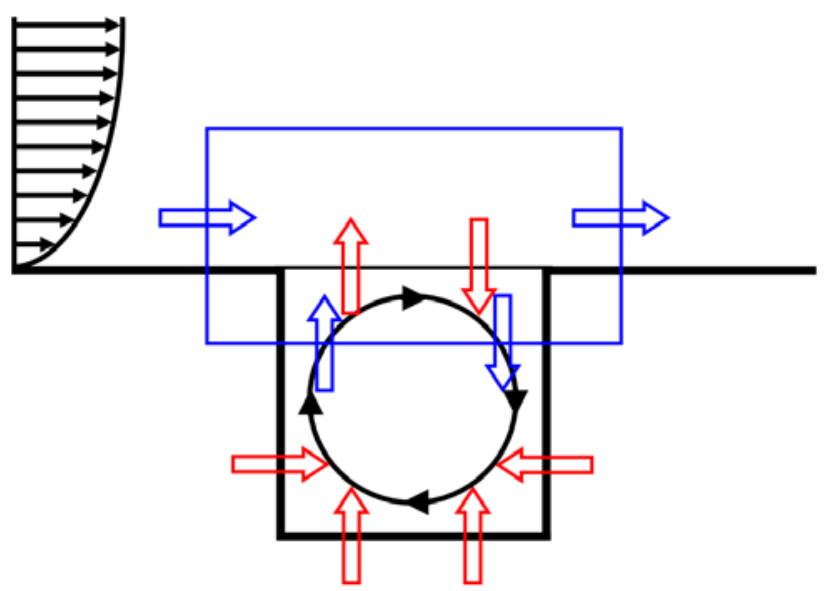

Figure 12: Sketch of the heat fluxes in a street canyon.

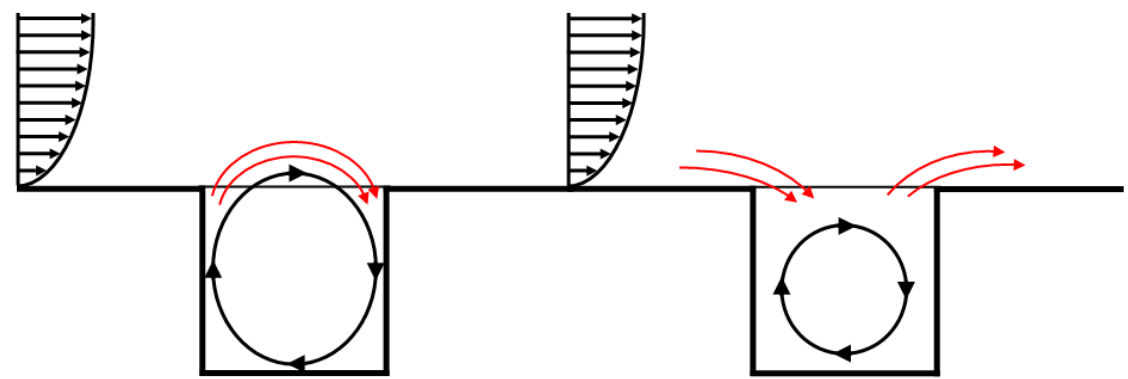

Figure 13: Sketch of the flow in street canyons with a main vortex with a diameter larger than the street canyon height (left) and smaller than the street canyon height (right). 
Tables

\begin{tabular}{llccc}
\hline & $70^{\circ} \mathrm{C}$ & $90^{\circ} \mathrm{C}$ & $110^{\circ} \mathrm{C}$ & $130^{\circ} \mathrm{C}$ \\
\hline $0.68 \mathrm{~m} / \mathrm{s}(\mathrm{Re}=9000)$ & 1.49 & 1.04 & 0.80 & 0.65 \\
\hline $1.07 \mathrm{~m} / \mathrm{s}(\mathrm{Re}=14200)$ & 3.68 & 2.58 & 1.99 & 1.62 \\
\hline $1.45 \mathrm{~m} / \mathrm{s}(\mathrm{Re}=19200)$ & 6.75 & 4.74 & 3.65 & 2.97 \\
\hline $1.86 \mathrm{~m} / \mathrm{s}(\mathrm{Re}=24600)$ & 11.11 & 7.79 & 6.00 & 4.88 \\
\hline $2.32 \mathrm{~m} / \mathrm{s}(\mathrm{Re}=30700)$ & 17.29 & 12.13 & 9.34 & 7.59 \\
\hline
\end{tabular}

Table 1: Froude numbers for the different surface temperatures and freestream velocities (with corresponding Reynolds numbers). 